\title{
ON NORMAL KUMMER FIELDS OVER A NON-MODULAR FIELD*
}

\author{
BY
}

\section{A. ADRIAN ALBERT}

1. Let $F$ be any non-modular field, $p$ an odd prime, $\zeta \neq 1$ a $p$ th root of unity. Suppose that $\mu$ in $F(\zeta)$ is not the $p$ th power of any quantity of $F(\zeta)$ so that the equation $y^{p}=\mu$ is irreducible in $F(\zeta)$. Then the field $F(y, \zeta)$ is called a Kummer $\dagger$ field over $F$.

In the present paper we shall give a formal construction of all normal Kummer fields over $F$. This is equivalent to a construction of all fields $F(x)$ of degree $p$ over $F$ such that $F(x, \zeta)$ is cyclic of degree $p$ over $F(\zeta)$. In particular we provide a construction of all cyclic fields of degree $p$ over $F$.

We shall also apply the cyclic case to prove that a normal division algebra $D$ of degree $p$ over $F$ is cyclic if and only if $D$ contains a quantity $y$ not in $F$ such that $y^{p}=\gamma$ in $F$.

2. The equation

$$
g(\xi) \equiv \xi^{p-1}+\xi^{p-2}+\cdots+\xi+1=0
$$

is irreducible in the field $R$ of all rational numbers and has all the primitive $p$ th roots of unity as roots. If $F$ is any non-modular field, then $g(\xi)$ has an irreducible factor $h(\xi)=0$ in $F$ and with $\zeta$ as a root. The roots of $h(\xi)=0$ are all powers of $\zeta$ and hence are in a sub-field $L$ of $R(\zeta)$. But then the coefficients of $h(\xi)=0$ are in $L$ so that the group of $h(\xi)$ with respect to $F$ is its group with respect to $L$. This latter group is the group of all the automorphisms of the cyclic field $R(\zeta)$ leaving the quantities of $L$ invariant and is a sub-group of the group of $R(\zeta)$. Every sub-group of a cyclic group is cyclic, so that $h(\xi)=0$ has a cyclic group generated by

$T$ :

$$
\zeta \leftarrow \zeta^{t},
$$

where $t$ is an integer belonging to the degree $n$ of $h(\xi)=0, t^{n} \equiv 1(\bmod p)$. We may write

$$
\zeta_{k}=\zeta^{t^{k-1}}, \quad \zeta_{n+1}=\zeta_{1}=\zeta^{\text {tn }} \quad(k=1, \cdots, n),
$$

so that we have

* This paper is a revision and amplification of the paper On cyclic equations of prime degree, which I presented to the Society on December 27, 1933; it was received by the editors March 17, 1934.

$\dagger$ If $F$ is the field of all rational numbers, then $F(y, \zeta)$ is the ordinary Kummer field of modern arithmetic. Our work is a generalization to any non-modular field of that special case. 


$$
\zeta_{k}=\zeta^{t_{k}}, t_{k} \equiv t^{k-1}(\bmod p), 1 \leqq t_{k}<p .
$$

Then $T$ is equivalent to the cyclic substitution $\left(\zeta_{1}, \zeta_{2}, \cdots, \zeta_{n}\right)$ on the roots of $h(\xi)=0$.

If $\lambda$ and $\mu$ are any two quantities of $K=F(\zeta)$ we say that $\lambda$ is $p$-equal to $\mu$ and write

$$
\lambda=\mu \text {. }
$$

(p)

H. Hasse* has then given a purely algebraic proof of

LEMMA 1. If

$$
y^{p}=\underset{(p)}{\neq} \neq 1
$$

then $Z=K(y)$ is cyclic of prime degree $p$ over $K$ and with generating automorphism

$S:$

$$
y \longleftrightarrow \zeta y .
$$

Conversely every cyclic field $Z$ of degree $p$ over $K$ is equal to a field $K(y)$,

$$
y^{p}=\mu \underset{(p)}{\neq 1}
$$

Moreover if also $Z=K(z), z^{p}=\mu^{\prime}$ in $K$, then

$$
\mu^{\prime}=\mu^{a} \text {, }
$$

(p)

so that $z=\lambda y^{a}$ where $\lambda$ is in $K$.

3. We now assume that $Z$ is any normal field of degree $p n$ over $F$ containing $K=F(\zeta)$ of degree $n$ over $F$. Then $K$ is the set of all quantities of $Z$ unaltered by a cyclic sub-group $H$ of $Z$ of order $p$ and $Z$ is cyclic of degree $p$ over $K$. By Lemma $1, Z=F(y, \zeta), y^{p}=\mu$ in $K$ and $H=\left(I, S, \cdots, S^{p-1}\right)$ where $S$ is given above. We can then decompose the group $G$ of $Z$ relative to $H$ and write $G=H+H \sigma_{1}+\cdots+H \sigma_{n-1}$. Then $I, \sigma_{1}, \cdots, \sigma_{n-1}$ carry $\zeta$ to the other roots of the irreducible equation $h(\xi)=0$. In particular one $\sigma_{i}=\tau$ carries $\zeta$ to $\zeta^{t}$.

We let $T=\tau^{p}$ so that $T$ also carries $\zeta$ to $\zeta^{t}$ since $t^{p} \equiv t(\bmod p)$. Then $\tau^{n}$ leaves $\zeta$ unaltered and is in $H$. Hence $\tau^{n}=S^{r}, T^{n}=S^{p r}=I$.

The group $G$ now has the decomposition $G=H+H T+\cdots+H T^{n-1}$. For otherwise $T^{r}=S^{i} T^{i}$ where $n>r>j$ so that $T^{r-g}=S^{i}$ leaves $\zeta$ unaltered, which is impossible. We have proved that

* Bericht über Klassenkörper, Jahresbericht der Deutschen Mathematiker-Vereinigung, vol. 36 (1927), pp. 232-311, p. 262. 


$$
G=\left(S^{i} T^{j}\right) \quad(i=0,1, \cdots, p-1 ; j=0,1, \cdots, n-1) .
$$

The group $G$ has a cyclic sub-group $\left(T^{i}\right)$ of order $n$ and hence $Z$ has a sub-field $F(x)$ of degree $p$ over $F$. Moreover

$$
y^{(r)}=\lambda y^{r} \quad(\lambda \text { in } K) .
$$

For $y^{(T)}$ in $Z$ evidently generates $K(y)$ and we may apply Lemma 1. But

$$
y^{(T S)}=\lambda \zeta^{r} y^{r}=y^{\left(S^{e} T\right)}=\zeta^{\circ} \lambda y^{r},
$$

where $e t \equiv r(\bmod p)$ so that $e \equiv r t^{n-1}(\bmod p)$. Hence $T S=S \cdot T$. Conversely if $T S=S^{\bullet} T$ then $r \equiv e t(\bmod p)$ is determined and we have proved*

THEOREM 1. Let $F(x)$ have degree $p$ over $F$ and $F(x, \zeta) \equiv Z$ be normal over $F$. Then $Z$ has the group

$$
S^{i} T^{i} \quad(i=0,1, \cdots, p-1 ; j=0,1, \cdots, n-1),
$$

such that $S^{p}=T^{n}=I$, the identity automorphism, and

$$
T S=S^{\bullet} T
$$$$
(0<e<p) \text {. }
$$

Moreover $Z=F(y, \zeta)$ where $y^{p}=\mu$ in $F(\zeta)$,

$$
\zeta^{(T)}=\zeta^{t}, y^{(T)}=\lambda y^{r}, \zeta^{(S)}=\zeta, \dot{y}(s)=\zeta y, \mu^{(r)} \underset{(p)}{=} \mu^{r},
$$

and $r \equiv e t(\bmod p)$.

Conversely every normal field $Z>F(\zeta)$ of degree $p^{n}$ over $K=F(\zeta)$ is generated as a field $Z=F(y, \zeta), y^{p}=\mu=\mu(\zeta)$ in $F(\zeta)$ such that

$$
\underset{(p)}{\neq 1}, \quad \mu\left(\zeta^{t}\right) \underset{(p)}{=} \mu^{r}
$$$$
(1 \leqq r<p) .
$$

The group of $Z$ is then given by (5), (6), (7) where $e$ is determined by $r \equiv e t(\bmod$ $p$ ) and $Z$ contains a sub-field $F(x)$ of degree $p$ over $F$, the field of all quantities of $Z$ unaltered by the automorphism $T$.

It is evident that $F(x)$ is uniquely determined in the sense of equivalence and is generated by any quantity

$$
x=\sum_{i=0}^{p-1} \alpha_{i}(\zeta) y^{i}=\sum_{i=0}^{p-1} \alpha_{i}\left(\zeta^{i}\right) \lambda^{i} y^{r i}
$$

for which at least one $\alpha_{i} \neq 0$ for $i>0$. Moreover the equation

$$
\phi(\eta) \equiv(\eta-x)\left(\eta-x^{(s)}\right) \cdots\left(\eta-x^{\left(s^{p-1}\right)}\right)
$$

has coefficients in $F$, is irreducible in $F$, and has $x$ as a root. Hence Theorem 1

\footnotetext{
- A similar result was obtained by Hilbert for the case $F=R$.
} 
gives a formal construction of all fields $F(x)$ of degree $p$ over $F$ with the property that $F(x, \zeta)$ is normal over $F$ in terms of the construction of all quantities $\mu$ satisfying (8).

If in particular $F(y, \zeta)$ has an abelian group, then $F(y, \zeta)=F(x) \times F(\zeta)$, where $F(x)$ is cyclic over $F$. Conversely if $F(x)$ is cyclic over $F$, then $F(x)$ $\times F(\zeta)=F(y, \zeta)$ has an abelian group, $e=1, r=t$ and we have

THEOREM 2. Let $\mu$ range over all quantities of $F(\zeta)$ such that

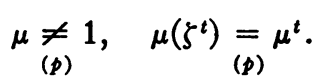

Then $Z=F(x) \times F(\zeta)$ where $F(x)$ is cyclic of degree $p$ over $F$. Conversely every cyclic field $F(x)$ of degree $p$ over $F$ is the uniquely defined sub-field of such an $F\left(\mu^{1 / p}, \zeta\right)$.

4. We proceed now to the construction of the quantities $\mu$. The condition

$$
\underset{(p)}{\mu \neq 1}
$$

is evidently an irreducibility condition depending intrinsically on $F$ itself and so must remain in our final conditions. We first prove

LEMMA 2. The integer $r$ satisfies the congruence

$$
r^{n} \equiv 1
$$

For

$$
\text { if } \mu^{(T)} \underset{(p)}{=} \mu^{r} \text { then } \underset{(p)}{\mu} \mu^{r n}
$$

and hence

$$
\mu^{r n-1}=1 .
$$

(p)

But then if $y^{p}=\mu$ the quantity $y^{r n-1}=\lambda y^{s}$ where $r^{n}-1 \equiv s(\bmod p), 0 \leqq s<p$ and $\lambda$ is in $F(\zeta)$. But $y^{s p}$ is then in $F(\zeta)$ so that $s=0$.

We have observed that $0<r<p$ so that there exists an integer $\rho$ such that

$$
\rho r \equiv 1
$$

We define

$$
\rho_{k} \equiv \rho^{k-1}(\bmod p), \quad 1 \leqq \rho_{k}<p,
$$

for all integer values of $k$, where $\rho_{n+1}=\rho_{1}=1$, and $\rho^{-\alpha}, \alpha>0$, is to be defined as a corresponding positive power of $\rho$. Then

$$
r \rho_{k} \equiv \rho_{k-1}
$$


We may then prove

LEMMA 3. Let $\lambda$ be any quantity of $F(\zeta)$ and define

$$
\mu=\prod_{k=1}^{n} \lambda\left(\zeta_{k}\right)^{\rho k} .
$$

Then

$$
\mu^{(T)}=\mu\left(\zeta^{t}\right) \underset{(p)}{=} \mu^{r} .
$$

For the automorphism $T$ carrying $\zeta$ to $\zeta^{t}$ carries each $\zeta_{k}$ to $\zeta_{k+1}$. Hence

$$
\mu^{(T)}=\prod_{k=1}^{n} \lambda\left(\zeta_{k+1}\right)^{\rho_{k}} \equiv \prod_{k=1}^{n} \lambda\left(\zeta_{k}\right)^{\rho_{k-1}},
$$

while, by (15),

$$
\mu^{r}=\prod_{k=1}^{n} \lambda\left(\zeta_{k}\right)^{r p_{k}} \underset{(p)}{=} \mu\left(\zeta^{t}\right)
$$

as desired.

Let now

$$
\mu\left(\zeta^{t}\right) \underset{(p)}{=} \mu^{r} \text { and } \mu \neq 1
$$

Then define

$$
M=\prod_{k=1}^{n} \Lambda\left(\zeta_{k}\right)^{\rho_{k}}
$$

where $\Lambda=\mu$. Then $\Lambda\left(\zeta_{k}\right)=\mu^{r k-1}$ so that

$$
\Lambda\left(\zeta_{k}\right)^{\rho_{k}} \underset{(p)}{=} \mu^{(r \rho) k-1} \underset{(p)}{=} \mu
$$

and hence

$$
M \underset{(p)}{=} \mu^{n}
$$

But $n$ is not divisible by $p$ so that $z=y^{n}$ generates $K(y)$,

$$
z^{p} \underset{(p)}{=} M .
$$

Hence $F(y, \zeta)=F(w, \zeta)$ where $w^{p}=M$ is a quantity of the form (16). Conversely if $\mu$ has the form (16) and

$$
\underset{(p)}{\neq} \neq 1
$$


then $F(y, \zeta), y^{p}=\mu$, is normal of degree $n p$ over $F$. We have proved

THEOREM 3. Let $\lambda$ range over all quantities of $F(\zeta)$ such that

$$
y^{p}=\mu \equiv \prod_{k=1}^{n} \lambda\left(\zeta_{k}\right)^{\rho_{k}} \underset{(p)}{\neq 1}
$$

Then $F(y, \zeta)$ is a normal field of Theorem 1. Conversely every normal field of Theorem 1 is generated by a $\mu$ defined by (22).

We have now succeeded in giving a formal construction of all the fields of Theorem 1. In particular we have constructed all cyclic fields of prime degree over $F$. For this case we have $\rho t \equiv 1(\bmod p)$, and may state

TheOREM 4. Let $\rho_{k} \equiv t^{p-k}(\bmod p)$ so that $t \rho_{k} \equiv t^{p-(k-1)} \equiv \rho_{k-1}(\bmod p)$ and let $\lambda$ range over all quantities of $F(\zeta)$ such that

$$
a=\prod_{k=1}^{n} \lambda\left(\zeta_{k}\right)^{\rho_{k}}
$$

is not the pth power of any quantity $b$ of $F(\zeta)$. Then if

$$
z^{p}=a,
$$

the field $F(z, \zeta)$ is cyclic of degree $n p$ over $F$ and

$$
F(z)=F(x) \times F(\zeta),
$$

where $F(x)$ is cyclic of degree $p$ over $F$. Conversely every cyclic field $F(x)$ of degree $p$ over $F$ is generated as the uniquely defined sub-field of such an $F(z, \zeta)$.

We have thus given a construction of all cyclic fields of prime degree over any non-modular field $F$ where the condition $a \neq b^{p}$ is the irreducibility condition.

5. On normal division algebras of degree $p$. Let $Z$ be a cyclic field of degree $p$ over $F$ so that every automorphism of $Z$ is a power of an automorphism $S$ given by $z \longleftrightarrow z^{S}$ for every $z$ and corresponding $z^{S}$ of $Z$. Define an algebra $D$ whose quantities have the form

$$
\sum_{i=0}^{p-1} z_{i} y^{i}
$$

such that

$$
y^{i} z=z^{s^{i}} y^{i}, \quad y^{p}=\gamma \neq 0 \text { in } F .
$$

Then $D$ is a cyclic algebra over $F$ and is a normal division algebra if and only 
if $\gamma \neq N(z)$ for any $z$ in $Z$. Evidently $D$ is uniquely defined by $Z, S, \gamma$ and we write

$$
D=(Z, S, \gamma)=(Z, S, \delta), \quad \delta=N(c) \gamma
$$

for any $c$ of $Z$. For $\gamma$ is replaced by $\delta$ when we replace $y$ by $c y$. Also*

$$
(Z, S, \gamma) \times(Z, S, \delta) \sim(Z, S, \gamma \delta) .
$$

If $D$ is a cyclic normal division algebra of degree $p$ over $F$, then $D$ has the above form and hence contains a sub-field $F(y), y^{p}=(\gamma)$ in $F$.

Conversely, let $D$ be any normal division algebra of degree $p$ over $F$ with $F(x), x^{p}=\beta$ in $F$ as sub-field. Let $K=F(\zeta)$ of degree $n$ over $F$. The algebra

$$
M=(K, T, 1),
$$

a cyclic algebra of degree $n$ over $F$, is a total matric algebra. We form the direct product $M \times D$ which evidently contains $K \times D=D_{0}$ as sub-algebra. Algebra $D_{0}$ is a normal division algebra of degree $p$ over $K$ and has the cyclic sub-field $Z=K(x)$. Moreover

$$
D_{0} \doteq(Z, S, \gamma),
$$

where $\gamma$ is in $K$ and the automorphism $S$ is given by the transformation

$$
y x=\zeta x y, \quad x s \equiv \zeta x .
$$

Let $M$ have a basis $\left(\epsilon^{i} j^{k}\right)(i, k=0,1, \cdots, n)$ such that $j^{n}=1$. Then in $D \times M$ we have

$$
j(y x) j^{-1}=y_{r} x=j(\zeta x y) j^{-1}=\zeta^{t} x y_{T},
$$

where $y_{T}=j y j^{-1}$ is in $D \times M$. But $y$ is commutative with $\zeta$ since $y$ is in $D_{0}$. Also $y \zeta=\zeta y$ implies that $y_{T} \zeta^{t}=\zeta^{t} y_{r}$ and hence $y_{r}$ is also commutative with $\zeta$. For $F\left(\zeta^{t}\right)=F(\zeta)$. The algebra of all quantities of $D \times M$ commutative with $\zeta$ is evidently $D_{0}$ so that $y_{r}$ is in $D_{0}$.

Since $y_{T} x=\zeta^{t} x y_{T}$ while $y^{t} x=\zeta^{t} x y^{t}$, we then have $y_{T}=d y^{t}$ where $d$ is in $Z$. Then

$$
\left(y_{T}\right)^{p}=j \gamma j^{-1}=\gamma\left(\zeta^{t}\right)=N(d) \gamma^{t},
$$

where $N(d)$ is the norm of the quantity $d$ of the cyclic field $Z$. But

$$
D_{0}^{t} \sim\left(Z, S, \gamma^{t}\right)=\left(Z, S, \gamma\left(\zeta^{t}\right)\right),
$$

by (33), (27).

* If $A$ is any normal simple algebra, then $A=M \times D$, where the total matric algebra $M$ and the normal division algebra $D$ are uniquely determined in the sense of equivalence. If $A$ and $B$ are two normal simple algebras with the same $D$, we say that $A$ and $B$ are similar, and write $A \sim B$. 
By applying (34) we have $D_{0} t^{2} \sim\left(Z, S, \gamma\left(\zeta^{t^{2}}\right)\right)$, and hence

$$
D_{0} t_{k} \sim\left(Z, S, \gamma\left(\zeta_{k}\right)\right)
$$

from which, if $u=\sum \rho_{k} t_{k}=n+\lambda p$ by (25),

$$
D_{0}{ }^{u} \sim D_{0}{ }^{n} \sim(Z, S, \alpha),
$$

where

$$
\alpha=\prod_{k=1}^{n} \gamma\left(\zeta_{k}\right)^{\rho k} .
$$

If $D$ is any normal simple algebra of prime degree $p$ over $F$, and $K$ is a field of degree $n$ not divisible by $p$, then $D$ is a total matric algebra if and only if $D \times K$ over $K$ is a total matric algebra. Moreover, if $r$ is prime to $p$, then $D^{r}$ is total matric if and only if $D$ is total matric. Hence, if $D_{0}=D \times K$ and $D_{0}{ }^{r}$ is a total matric algebra, then so is $D$.

Algebra $D_{0}{ }^{n}$ is a normal division algebra since $D$ is a normal division algebra. Hence $\alpha \neq N(c)$ for any $c$ of $Z$. In particular $\alpha \neq b^{p}$ for any $b$ of $K$. Thus $D_{0}$ contains a cyclic field* $W$ of prime degree $p$ over $F$. But then $D_{0}{ }^{n} \times W^{\prime}$ over $W^{\prime} \cong W_{K}$, the composite of $W$ and $K$, is a total matric algebra. Hence $D_{0} \times W^{\prime}$ is a total matric algebra and. so must be $D \times \bar{W}$ over $\bar{W}, \bar{W} \cong W$. But then $D$ has a sub-field equivalent to $W$ and is cyclic.

Theorem 5. A normal division algebra $D$ of prime degree $p$ over $F$ is cyclic if and only if $D$ has a sub-field $F(x), x^{p}=\gamma$ in $F$.

* The cyclic sub-field of $F\left(\alpha^{1 / p}\right)$ defined by Theorem 4 .

The Institute for Advanced Study,

Princeton, N. J. 